\title{
La ecología como práctica educativa interdisciplinar entre educación física y tecnología ${ }^{1}$
}

\author{
Márjory Cuervo Bernal² \\ Universidad Pedagógica Nacional, Bogotá, Colombia \\ macube92@hotmail.com \\ Alejandra Gutiérrez Rodríguez ${ }^{3}$ \\ Universidad Pedagógica Nacional, Bogotá, Colombia \\ alejaguti3163@gmail.com
}

1 Artículo de reflexión

2 Estudiante Licenciatura en Diseño Tecnológico Noveno Semestre. Universidad Pedagógica Nacional

3 Estudiante Licenciatura en Diseño Tecnológico Noveno Semestre. Universidad Pedagógica Nacional. 


\section{La ecología como práctica educativa interdisciplinar entre educación física y tecnología}

\section{Resumen}

Este artículo pretende mostrar cómo la problemática actual del medio ambiente se puede abordar desde diferentes áreas del conocimiento, a partir de la necesidad de entender la interdisciplinariedad en el aula como factor trascendental para que el niño genere propuestas en su entorno. La ecología será esa área del conocimiento que permitirá la articulación de la educación física y la tecnología buscando aportar a que la educación sea entendida como relación de las realidades de la sociedad y la comunidad del niño.

Palabras clave: Ecología, tecnología, educación física, medio ambiente, interdisciplinariedad.

\section{Ecology as an interdisciplinary educational practice. Between physical education and technology}

\section{Abstract}

This article tries to show how a current problematics like it is the environment can generate a boarding from different areas of the knowledge. Considering to be like that the need to understand the interdisciplinary in the classroom as transcendental factor in order that the child generates proposed level of his environment. For which the ecology will be this area of the knowledge that will allow the joint of the physical education and the technology seeking to each to that the education is understood as the relation of the realities of the company and the community of the child.

Key words: Ecology, technology, physical education, environment, interdisciplinary.

\section{A ecologia como prática educativa interdisciplinar entre educação física e tecnología}

\section{Resumo}

Este artigo pretende mostrar como a problemática atual do meio ambiente se pode abordar desde diferentes áreas do conhecimento, a partir da necessidade de entender a interdisciplinariedad no sala como factor trascendental para que o menino gere propostas em seu meio. A ecologia será essa área do conhecimento que permitirá a articulação da educação física e a tecnologia procurando contribuir a que a educação seja entendida como relação das realidades da sociedade e a comunidade do menino.

Palavras-chave: Ecologia, tecnologia, educação física 


\section{Introducción}

La educación y la búsqueda de la conciencia ambiental deben generar prácticas que estén en relación con la realidad más cercana de las y los sujetos, en donde el desarrollo integral de contenidos posibiliten que la problemática ambiental se visibilice desde factores socialmente relevantes que busquen justicia, igualdad y respeto, mejorando la calidad de vida sin dañar el entorno físico y lo que allí se encuentra.

La educación física y la tecnología son susceptibles de integrarse desde la ecología mediante experiencias interdisciplinares que se basan en las relaciones activas dadas entre las dos disciplinas, buscando que la problemática ambiental planteada desde la apropiación de los recursos naturales y desechos forme caminos de igualdad y armonía entre el ser humano y lo que le rodea:

No podemos olvidar que el aprendizaje a través del movimiento es un método de enseñanza milenario. Cuando se utiliza la actividad motriz como medio para aprender, la interacción entre el niño y la materia es más completa y se incrementa la posibilidad de su implicación activa (Méndez et al., 2009).

Se defiende la educación física como parte del desarrollo interdisciplinar que se busca en la educación ambiental a través de la ecología. La tecnología busca que el ser humano aborde "situaciones problemáticas que trascienden los límites de una disciplina concreta para detectar, analizar y solucionar problemas nuevos con los que jamás se habían encontrado" (Rodríguez, s.f), de modo que la realidad y la vida misma son partícipes en la formación integral del ser humano, en donde los procesos mentales que se dan desde la interacción humana propician la reflexión sobre las situaciones problemas a las que se les busca dar solución a través de la tecnología.

Este artículo se divide en tres partes. La primera consta del planteamiento de la problemática ecológica actual, la sobreexplotación de los recursos naturales y las dinámicas de predominio del hombre sobre la naturaleza. Asimismo, examina cómo esto se ha traducido en la reducción de las relaciones de los sujetos consigo mismos, con los demás y con el medio. Se problematiza que los avances tecnológicos han tratado de facilitar la vida de las personas al punto de limitar sus movimientos y las relaciones que se generan, además, se evidencia el papel que ha tenido la escuela para fortalecer estas dinámicas ya que no ha tenido una intervención fuerte en cuanto a la integración de los contenidos y los saberes para la mitigación de las problemáticas ecológicas.

La segunda parte consta de la contextualización de lo ecológico y aborda la pregunta por su papel como disciplina para integrar a otras, se ofrece una mirada 
holística del conocimiento para generar modos de vida armónicos en cuanto a las relaciones del sujeto, su cuerpo y su ser, con los demás y con el medio en el cual están inmersos. A partir de esto se observa el aporte que dan específicamente las disciplinas de educación física y tecnología para la formación de un modo de vida ecológico. En una tercera y última parte se plantea a modo de conclusión la contextualización pertinente al tema de interdisciplinariedad con el objetivo de dar algunas definiciones sobre la integración de las dos disciplinas y la importancia de esta en la búsqueda de relaciones armónicas entre ser humano-naturaleza y la implicación de una mirada holística para conservar la vida y mantener unas dinámicas de vida digna.

\section{La problemática ecológica}

La contaminación ambiental es un tema que hoy en día compete a todos y todas las especies vivientes que habitan en el planeta tierra. Guattari (1990) dice "el planeta Tierra vive un periodo de intensas transformaciones técnico-científicas como contrapartida de las cuales se han engendrado fenómenos de desequilibrio ecológico que amenazan, a corto plazo, si no se le pone remedio, la implantación de la vida sobre su superficie". La relación que ha tenido el hombre con la naturaleza ha sido de predominio sobre ella, generando una visión dualista entre hombre-naturaleza y a su vez convirtiéndola en un objeto o una herramienta que está dispuesta simplemente para servirnos como amos del planeta.

Esto se evidencia a partir de "los patrones dominantes de producción y consumo que están causando devastación en la naturaleza, agotamiento de recursos y una extinción masiva de especies. Las comunidades están siendo destruidas. Los beneficios del desarrollo no se comparten equitativamente y la brecha entre ricos y pobres se está ensanchando" (Carta de la Tierra, 2000). Desde la aparición de las industrias comienza un desarrollo tecnológico que devasta con lo que esté a la mano para satisfacer las necesidades del hombre. La tecnología ha estado siempre inmersa en el desarrollo de la humanidad, pues, históricamente el hombre ha buscado los modos de sobre vivir al punto de facilitar su desenvolvimiento cotidiano, pero en esta búsqueda ha acabado con todo lo que a su alrededor encuentra, incluyendo a los de su misma especie. Esto genera una verdadera preocupación por la sustentabilidad del ambiente para la preservación de todas las especies vivientes.

No podríamos culpar del todo a las recientes generaciones por esta crisis ya que desde el inicio de la historia conocida, el ser humano y los sistemas de producción que éste ha creado han hecho que el medio en donde vive se esté acabando incontroladamente, generando gran cantidad de desequilibrios entre sus sistemas físicos $\square$ luz, temperatura, humedad, aire, atmósfera $\square$, biológicos $\square$ plantas, animales y en general todos los seres vivos dentro de la naturaleza , sociales, culturales, económicos y políticos. 


\section{Cuerpo, tecnología y sociedad}

El modelo capitalista actual mantiene las dinámicas de un progreso fundamentado en la eliminación de todo lo natural para sustituirlo por lo artificial, no sólo hablando desde lo biológico o lo ambiental, pues, el sujeto como individuo ha venido reduciendo sus posibilidades de relación consigo mismo y, por otro lado, algunos avances tecnológicos le han quitado el papel al cuerpo como herramienta primordial para el desarrollo de variadas tareas en la vida cotidiana; se reduce el concepto de tecnología al artefacto físico y sus modificaciones, llevando al hombre a buscar esquemas sociales de materialismo y consumismo que buscan llevar al olvido que el cuerpo es el primer recurso tecnológico.

Las necesidades han trascendido al punto de convertirnos en sujetos de consumo, aquellas ya no se limitan simplemente a necesidades vitales como alimentarse y estar en condiciones óptimas de salud, o la necesidad de generar relaciones sociales; ahora las necesidades han pasado a un plano más complejo en donde los últimos avances tecnológicos han facilitado en gran medida las funciones básicas que se desarrollan en la cotidianidad, pero, que aun así han generado gran dependencia llegando al punto de que el ser humano destruye su naturalidad y la naturaleza que le rodea. Una causa de esto es la instrumentalización que el hombre ha implantado sobre la naturaleza, sus dinámicas de vida son dirigidas hacia el aprovechamiento, utilización y explotación irresponsable de ésta, de sus recursos y sus especies, incluyendo al hombre. Es la dominación que ha existido de algunos seres humanos sobre otros durante la historia lo que remite a la concepción de que el cuerpo se ha convertido en simple objeto de trabajo y explotación, desde diferentes ámbitos de la vida. La tecnología y el hombre desde sus actividades diarias, y el cuerpo como herramienta fundamental para el desarrollo, han hecho de la vida una forma insostenible.

\section{La interdisciplinariedad en la escuela}

La escuela ha sido un lugar de segregación en donde cada disciplina se desarrolla de manera independiente sin tener en cuenta las necesidades de una sociedad que busca el cambio para dejar de lado la educación tradicional que no está en contexto y que no permite. La educación en ecología parte de la identificación de los problemas que se dan a partir de las relaciones que tiene el ser humano con los demás plantas y animales para encontrar otras relaciones en cuanto a lo económico, político, social y cultural, que han sido ámbitos que definen la forma de vida del ser humano. Una vez encontradas estas relaciones se buscan los problemas que se derivan de estas para finalmente buscar la mejor solución desde la integración completa de saberes, dando una mirada global a la aplicación interdisciplinaria de la ecología en la educación de hoy en día. 
A continuación se abordará la ventaja del estudio de la ecología como práctica educativa que integra diversos saberes que contribuyen a que en la educación se logre la interdisciplinariedad específicamente desde la resolución de problemas frente al cuidado de los recursos que tenemos y el aprovechamiento de desechos; en este caso es fundamental para el trabajo conjunto de la tecnología y la educación física apostando que la educación ambiental en ecología sea parte de un currículo formal o no formal en el campo educativo.

\section{La propuesta ecológica}

La ecología es la ciencia que estudia las interacciones de un individuo con otros y con su ambiente físico. Estas interacciones determinan la distribución y la abundancia de los organismos en un área particular y en la superficie terrestre" (Parga y Cárdenas, 2013). La ecología busca a partir de las distintas disciplinas y áreas de conocimiento generar conciencia por lo que se tiene para su preservación y buen uso.

La anterior es una aproximación básica de la ecología, que como práctica educativa busca que todas las relaciones en las que el ser humano y demás especies animales y vegetales $\square$ sean vistas y planteadas desde la interdisciplinariedad de las áreas básicas del sector educativo para la formación y transformación de un ser humano integral con miras a que éste mantenga unas relaciones de armonía con el medio en el que vive.

Todo el funcionamiento de la naturaleza y las estructuras que allí se forman son las bases de una educación ambiental en la que el ser humano interviene de manera directa, modificando cada sistema dentro de su medio a partir de procesos tecnológicos que le permiten aplicar su conocimiento y de manera directa contribuir a utilizar el cuerpo como un medio y no como un fin, siendo este fin la máxima capacidad del ser humano para preservarse y preservar lo que su entorno le brinda.

Parafraseando a Gudynas y Evia (1995), el ambiente es un sistema que presenta elementos interactuantes y que determinan aspectos de la estructura, función y comportamiento del ser humano, precisamente son estos aspectos los que busca la educación ambiental, pero que sólo a partir de la ecología se pueden tratar específicamente.

Es por lo planteado anteriormente que se busca la aplicación de la ecología, basada en la construcción y apropiación de hábitos que permiten al ser humano aportar a la solución de problemas ambientales como la contaminación y la escasez de recursos naturales que generan devastación y degradación en el medio ambiente, se da en contextos de precarización de lo nuestro, en donde la única salida es conservar y preservar, desde las distintas prácticas que el contexto brinde y desde las 
apreciaciones que la educación, como fuente de conocimiento, brinde a la sociedad y el espacio en el que se habita.

\section{Interdisciplinariedad}

La interdisciplinariedad es entendida como "la comprensión y solución colaborativa de problemas sociales reales. El conocimiento interdisciplinar establece unas metas que, a través de unos medios teórico-prácticos, pretende buscar soluciones razonables y justificables a cuestiones que están más allá de los límites de una disciplina" (Fernández, 2010). Es de importancia tomar en cuenta disciplinas como la educación física y la tecnología para abordar problemáticas ambientales, puesto que ello permite generar respuestas a nivel crítico y analítico con mayor solidez y veracidad.

En cuanto a la educación física y tecnología como áreas fundamentales en la educación formal y no formal, se aclara que son dos disciplinas que en la escuela se tornan diferentes en cuanto a la práctica y la cátedra respectivamente. Partiendo de esto, la tecnología busca generar en el estudiante reflexiones y pensamientos que le permitan planear y diseñar alternativas de solución a necesidades que encuentre en su contexto. Por otro lado, la educación física busca que desde la práctica el estudiante dé solución a problemas que se dan desde su corporeidad y su motricidad para el desarrollo de habilidades y capacidades, permitiendo que el estudiante reflexione y proponga acciones que lleguen a una respuesta planeada desde estrategias y basadas en su autonomía. En este sentido, tanto la educación física como la tecnología buscan dar solución a problemas; desde la ecología se pretende dar solución a los problemas ambientales que tanto atañe hoy en día a la humanidad y que se hace importante desde las prácticas concretas que buscan la preservación de la vida en el planeta tierra.

Las relaciones que se dan en las prácticas educativas formales o no formales deben buscar la integración de contenidos, sin embargo, la escuela se ha encargado de mantener aisladas cada una de las materias, en donde la repetición cobra vital importancia y la transmisión de conocimiento es una estrategia para privar al sujeto de adquirir su propio conocimiento. Por otro lado, en lo que respecta a la temática ambiental se busca que la interdisciplinariedad sea la base para fomentar una educación ambiental en la que los y las estudiantes sean capaces de reconocer su entorno como propio, cuidarlo y conservarlo para mantener la existencia humana.

Noguera (2009), desde la filosofía ambiental, nos remite a redescubrir nuestro papel en la vida como seres y cuerpos que son naturaleza, en donde pasemos de "una actitud de dominio del hombre sobre la naturaleza a una actitud de reconocernos como naturaleza en expansión y reconocer que todo lo que hacemos (arte, técnica, tecnología, ciencia y, en general, cultura) es expansión de nuestro cuerpo que es 
naturaleza". Para entendernos como parte emergente de la naturaleza y no como algo aislado a ella, ya que tratar de objetivarla, cuantificarla y haberla explotado de manera absurda, nos ha llevado a la actual crisis del ser, alejándonos y perdiendo profundamente lo que nos hace ser humanos: la naturaleza. 


\section{Referencias}

Carta a la tierra (2000). Recuperado de: http://earthcharter.org/invent/images/uploads/ echarter_spanish.pdf

Erazo, M y Cárdenas, R. (2013). Ecología: Impacto de la problemática ambiental actual sobre la salud y el ambiente. Colombia: Ecoe Ediciones.

Fernández, R. Luis. (2010). Interdisciplinariedad en la construcción del conocimiento: ¿Más allá de Bolonia? Innovación educativa, 20, 157-166.

Guattari, F. (1996). Las Tres Ecologías. Francia: Galilée

Gudynas, E. y Evia G. (1993). Ecología social: manual para educadores populares. Colombia: Magisterio.

Noguera, P. y Pineda. A. (2009). Filosofía ambiental y fenomenología: el paso del sujetoobjeto a la trama de vida en clave de la pregunta por el habitar poético contemporáneo. Acta Fenomenológica Latinoamericana III (Actas del IV Coloquio Latinoamericano de Fenomenología): 261-277.

Rodríguez, A. (1998). Ciencia, Tecnología y Sociedad: una mirada desde la Educación en Tecnología. Revista Iberoamericana de educación 18, 107-144.

Recibido: 9 de septiembre 2015

Aceptado: 30 de noviembre 2015

Cómo citar: Bernal, M y Gutiérrez, A. (2016) La ecología como práctica educativa interdisciplinar entre educación física y tecnología.

Praxis Pedagógica, n. ${ }^{\circ} 18,91-99$ 